\title{
JURISPRUDENCIA AMBIENTAL EN EL PAÍS VASCO (PRIMER SEMESTRE 2018)
}

\author{
IÑIGO LAZKANO BROTÓNS \\ Profesor colaborador \\ Universidad del País Vasco / Euskal Herriko Unibersitatea
}


Sumario: 1. Problemas jurídicos relativos al establecimiento de la incineradora en Gipuzkoa. 2. Evaluación ambiental estratégica y modificación puntual de planes generales. 3. Responsabilidad patrimonial municipal por omisión de control de ruidos ocasionados por particulares. 4. Sanciones administrativas por ocupación del demanio costero y por acampada en playas. 5. llegalidad de sanción impuesta a centro de salud público por falta de comunicación previa de actividad clasificada. 6. Gastos de descontaminación del suelo y reparcelación urbanística.

\section{PROBLEMAS JURÍDICOS RELATIVOS AL ESTABLECIMIENTO DE LA INCINERADORA EN GIPUZKOA}

La STSJPV 499/2017, de 29 de diciembre (sala de lo contenciosoadministrativo, sección primera, ponente: Murgoitio Estefanía), desestima el recurso de apelación interpuesto contra la sentencia de instancia que había anulado los acuerdos del Consorcio de Residuos de Gipuzkoa, que habían aprobado la resolución por mutuo acuerdo entre la unión temporal de empresas contratista y la sociedad anónima Gestión de Residuos de Gipuzkoa (entidad privada creada por el citado Consorcio) del contrato destinado a la construcción, puesta en marcha y explotación del Centro de Gestión de Residuos de Gipuzkoa (que, junto a otras instalaciones, incluía la incineradora), al calificarlos como desviación de poder. En una sentencia compleja, en el que las posturas de recurrentes y demandados, apelantes y apelados, constituyen grupos fluctuantes de antagonistas en función de su composición, alterada por los diversos resultados electorales (al tratarse, en bastantes casos, de mancomunidades locales de servicios), el TSJPV va a ratificar el fallo de la sentencia de instancia, si bien matizando las argumentaciones.

En primer lugar, y en esto no hay discrepancias entre la sentencia de instancia y la de apelación, se reconoce la competencia jurisdiccional contenciosoadministrativa en relación a la materia (cuestionada por los apelantes, algo que -por cierto- no hicieron en el recurso originario). Los consorcios son entes de derecho público capaces de gestionar los servicios que tuvieran encomendados valiéndose de las formas establecidas para las administraciones consorciadas (foral y local en este caso). La creación de la sociedad anónima mercantil instrumental y su calificación como medio propio y servicio técnico, para ser el soporte de una encomienda de gestión con el objetivo de llevar a cabo dicho proyecto, no hacen posible concebir el hito de la 
extinción del contrato como una decisión autónoma de la sociedad anónima instrumental que pueda prevalecer con sustantividad propia respecto de la voluntad rectora y principal del consorcio. Esto es así porque la encomienda de gestión a un medio propio, sin mediar contratación pública entre ambos entes y conforme a la doctrina de los encargos in house del Derecho europeo, solo pudo atribuir a dicho medio o ente instrumental cometidos materiales, técnicos o de servicios prestados al ente creador, pero sin cesión de la titularidad de la competencia, ni de los elementos constitutivos de su ejercicio. Por ello es la voluntad del consorcio, mediante su asamblea (órgano real), la que se traduce y refleja de modo automático como voluntad de la junta de accionistas de la sociedad anónima instrumental (órgano nominal y no efectivo) como voto unánime, y por tanto, no cabe sostener la ficción inversa de que quien decide es la junta de accionistas en régimen de derecho privado. El acuerdo asambleario estaba, en consecuencia, sujeto a Derecho administrativo, por más que tuviese por objeto material decidir sobre la extinción de un contrato de derecho privado.

Por otra parte, el TSJPV mantiene que el acuerdo asambleario adoptado incurre en desviación de poder. Pero no por el motivo que señala la sentencia de instancia, contradecir la decisión de las Juntas Generales de Gipuzkoa que normativamente habían aprobado la necesidad de la implantación del centro de gestión de residuos de Gipuzkoa como sistema integral de gestión del Territorio Histórico, sino por otra causa. Hay que valorar si la extinción por mutuo acuerdo del contrato se produjo en el marco estricto de observancia de los fines constitutivos del consorcio, con objeto de dar una nueva e idónea dimensión a la infraestructura cuya realización se le había encomendado y evitar así los sobrecostes para las mancomunidades consorciadas, para posteriormente volver a contratar su ejecución y explotación sin cuestionar las previsiones y determinaciones del planeamiento sectorial o territorial; o si, por el contrario, la suspensión de la ejecución de la obra y posterior resolución indemnizada pactada con la UTE contratista, solo respondió a la intención de inejecutar definitivamente la incineradora y sustituirla por otras infraestructuras alternativas ajenas a las previsiones y al encargo atribuido al consorcio. Esta última conducta es la que aprecia el TSJPV, a la luz de actuaciones anteriores, 
coetáneas y posteriores implementadas por el consorcio y su sociedad gestora, incluyendo las precisas menciones sobre el contexto público y medial en que la decisión se adoptó, mediante informaciones urbi et orbe acerca de que la referida incineradora no iba a ser construida (en notas de prensa, folletos explicativos, comparecencias públicas y entrevistas). Concurre, a su juicio, desviación de poder, pues las razones que se explicitaban y trataban de dar justificación aparente de interés público a la extinción del contrato (que sería así solo coyuntural y puramente técnica), no se correspondían con las verdaderas finalidades de la decisión administrativa, de carácter estructural, definitivo y de radical y completo apartamiento del modelo al que dicho contrato se ajustaba.

La STSJPV 361/2017, de 27 de septiembre (sala de lo contenciosoadministrativo, sección primera, ponente: Fernández Fernández), ratifica en apelación la de instancia que había desestimado el recurso interpuesto por una asociación (Gurasos Elkartea) que reclamaba a la Diputación Foral de Gipuzkoa la apertura de un proceso de deliberación participativa relacionado con la decisión de construir la planta incineradora de Zubieta. Para el TSJPV la asociación apelante presupone la integración del derecho de participación en materia ambiental (protegido por el Convenio de Aarhus, varias directivas comunitarias y la normativa interna estatal y foral de transposición) en el derecho de participación política del art. 23.1 CE, lo que es contradictorio con la jurisprudencia constitucional. La recurrente ha instado la tutela de un derecho, el del art. 23.1 CE, que no le corresponde, sino, en su caso, a las personas físicas. Derecho que no debe ser confundido con el de audiencia o participación de los interesados en los procedimientos de aprobación de los actos administrativos (art. 105.c CE), que sí correspondería a la asociación, pero que no puede ser canalizado a través de un procedimiento judicial en protección de los derechos fundamentales..

\section{EVALUACIÓN AMBIENTAL ESTRATÉGICA Y MODIFICACIÓN PUNTUAL DE PLANES GENERALES}

La impugnación de la aprobación definitiva de la modificación del plan general de ordenación urbana (PGOU) de Donostia-San Sebastián, referida a dos ámbitos urbanísticos muy concretos (AU05 Ategorrieta y NU02 Ulia) es la que 
motiva el dictado de la STSJPV 434/2017, de 29 de septiembre (sala de lo contencioso-administrativo, sección segunda, ponente: Alberdi Larizgoitia). El recurrente basa su impugnación en tres argumentos: la falta de motivación de la conveniencia de la modificación, el incumplimiento del deber de adaptación a los estándares dotacionales locales previstos normativamente y la omisión del procedimiento de evaluación ambiental simplificada. EI TSJPV va a estimar el recurso solamente en base al tercero de los motivos.

En lo fundamental, la nueva ordenación que pretendía adoptarse preveía en ambas zonas cambiar el régimen de usos preexistente (básicamente viviendas de promoción libre) por equipamientos públicos en la modalidad de alojamientos dotacionales o residencias para jóvenes. La motivación municipal dada a esa modificación se estima suficiente, según el TSJPV, al estar basada en la proporción del número de solicitantes de alojamientos dotacionales (10 a 1 , en favor de los jóvenes, aunque los datos de la sentencia son contradictorios). El reproche de la falta de estudio de alternativas para la ubicación del equipamiento y la opinión de que se trata de un ámbito más apropiado para la localización de un parque público, no tienen la entidad necesaria para determinar la anulación del acuerdo recurrido. Desestima el TSJPV la invalidez de la modificación por incumplimiento del deber de adaptación a los estándares dotaciones locales, ya que no se alega ni acredita que la ordenación modificada infrinja tales estándares, lo que no parece evidente -señala el órgano judicial- teniendo en cuenta que sustancialmente reduce la edificabilidad residencial y aumenta los equipamientos públicos.

El elemento central de la sentencia, y lo que en definitiva motiva la estimación del recurso y la anulación del acto recurrido, es la admisión de que se ha omitido el necesario procedimiento de evaluación ambiental simplificada. La argumentación del TSJPV, no obstante, plantea ciertas dudas. La sentencia parte del análisis de la situación legislativa en la CAPV: la Ley 3/1998 General de Protección del Medio Ambiente y el Decreto 211/2012 que desarrolla el procedimiento de evaluación ambiental estratégica de planes y programas no se hallan adaptados a la estatal Ley 21/2013, de Evaluación Ambiental. Por ello, y basándose en la más reciente jurisprudencia del Tribunal Constitucional, el TSJPV, en cuanto que operador jurídico, inaplica la ley autonómica en virtud 
del principio de prevalencia y procede a aplicar directamente la vigente legislación estatal sobre evaluación ambiental. Llega entonces a la conclusión de que, en base al art. 6.2.a) de la Ley 21/2013, modificaciones menores (como las aquí analizadas) de planes y programas sujetos a evaluación ambiental ordinaria deben ser objeto de evaluación ambiental simplificada, so pena de nulidad (como ha ocurrido en este caso). Pero el argumento no es tan simple en la práctica. El TSJPV afirma que ello se debe a que un PGOU "por definición, dado su carácter de planeamiento general, establece el marco para la futura autorización de proyectos legalmente sometidos a evaluación de impacto ambiental y, además, tiene por objeto la ordenación de usos del suelo". Pero esa afirmación no debe hacerse así, en abstracto, sino analizando en concreto si la nueva ordenación que se aprueba puede amparar un proyecto que esté sometido en el futuro a evaluación de impacto ambiental. Y para ello la ordenación de usos del suelo prevista en la modificación, la superficie afectada y la ubicación de lo ordenado se estiman fundamentales. Si no, cualquier modificación del PGOU, por nimia que fuera, debería ser objeto de evaluación ambiental simplificada, algo que no parece lógico ni responde a las previsiones de la normativa de evaluación. Por eso mismo no estaba descaminada la alegación del recurrente (calificada como error en la sentencia) que exigía dicho tipo de evaluación en la medida en que el (futuro) proyecto de urbanización que la modificación entrañaba se encontrase incluido en uno de los apartados del Anexo II de la Ley 21/2013 (relativo a proyectos sometidos a evaluación de impacto ambiental simplificada: proyectos situados fuera de áreas urbanizadas de urbanizaciones y que en superficie ocupen más de una hectárea), pero nada de eso se discute en la resolución judicial y los (limitados) datos de hecho que la sentencia proporciona respecto a las superficies afectadas por la modificación no permiten saber si ese supuesto se incluye en las previsiones legales referidas.

Hay varias sentencias en relación a las exigencias medioambientales que han de cumplirse para la aprobación definitiva de planes urbanísticos. Las sentencias del TSJPV 418/2017 y 419/2017, de 22 de septiembre (sala de lo contencioso-administrativo, sección segunda, ponente: Alberdi Larizgoitia), desestiman los recursos imntrepuestos contra la aprobación definitiva del plan 
especial de ordenación urbana de una concreta unidad de ejecución en el municipio de Berango. La omisión del estudio de impacto acústico o de informe de la administración hidráulica se desestiman como argumentos impugnatorios por motivos exclusivamente temporales: las normas que los exigían no podían aplicarse a planes ya aprobados inicialmente (como en el caso de referencia). Tampoco se hallaría sometido a una evaluación ambiental estratégica, pues la normativa autonómica entonces en vigor (no la Ley 21/2013 de evaluación ambiental, cuya vigencia es posterior) excluía de su ámbito este tipo de planes especiales. La falta de exposición al público de un resumen ejecutivo de las alteraciones de la ordenación (previsto en la normativa reglamentaria de desarrollo de la Ley 2/2006 de Suelo y Urbanismo del País Vasco) se considera una exigencia eminentemente formalista, habida cuenta del limitado alcance del plan especial impugnado, y de escasa trascendencia o relevancia.

\section{RESPONSABILIDAD PATRIMONIAL MUNICIPAL POR OMISIÓN DE CONTROL DE RUIDOS OCASIONADOS POR PARTICULARES}

En esta materia hay que destacar la STSJPV 528/2017, de 20 de septiembre (sala de lo contencioso-administrativo, sección tercera, ponente: Gonzalez Saiz). La sentencia ha de resolver el recurso de apelación interpuesto contra la del juzgado de lo contencioso-administrativo que condenó al Ayuntamiento de Mutriku a resarcir a los recurrentes en concepto de responsabilidad patrimonial derivada del defectuoso funcionamiento del servicio público consistente en no haber adoptado durante largos años medida alguna para evitar que se sucedieran los ruidos que, procedentes de un local de hostelería colindante, excedían en su intensidad de los límites normativamente establecidos. La sentencia es un ejemplo de claridad en cuanto a su estructura interna y a su propia redacción. En ella se afirma que: a) al ser una acción de responsabilidad patrimonial de una administración pública, la única destinataria de la demanda es ésta, en tanto que titular del servicio público defectuosamente atendido, sin que proceda haber demandado también al titular del negocio causante del ruido; b) el valor probatorio que se otorga al informe técnico de la sociedad limitada privada que lo elabora deriva tanto del propio reconocimiento que al mismo dió la recurrente en el procedimiento administrativo previo como del hecho de ser elaborado a instancia del órgano foral competente para la 
adopción de medidas correctoras en relación a la licencia de actividad (lo que acredita la calidad técnica y la confianza que dicha empresa privada generaba), aunque no se presentara específicamente el citado informe como prueba pericial en el proceso judicial; c) la relación de parentesco o amistad de determinados testigos presentados por la recurrente no priva de eficacia a su testimonio, el cual ha de ser valorado, como el de los testigos presentados por la demandada, en función de su grado de objetividad y a su coherencia interna con el resto de pruebas y los actos propios de las partes enfrentadas; d) la fecha de inicio de los ruidos puede ser determinada en base al momento de apertura del establecimiento, pues ya en la fase de alegaciones seguido en el procedimiento de obtención de la licencia, cuando el local iba a reformarse, se manifestó la defectuosa o nula insonorización preexistente que ya generaba molestias; e) no es necesario que el ruido haya lesionado efectivamente la salud de las personas afectadas, sino que basta con que les haya impedido utilizar su vivienda en términos de razonable tranquilidad, para cuya determinación pueden tenerse en consideración factores como la superación de los límites normativamente impuestos, la intensidad, los horarios en que se produce, la duración temporal, etc.; y f) la cuantificación de la indemnización por daños fijada por el tribunal de instancia no parece desproporcionada ni irrazonable (aunque su cuantía no se cita expresamente en la sentencia), a la luz de los criterios que han de regir en esta materia. Este último aspecto merece una especial atención.

En efecto, la sentencia referida elabora detalladamente una serie de criterios para la determinación de la cuantía de la indemnización en este tipo de casos. En principio, más allá de los daños puramente materiales, la utilización del domicilio en términos de razonable tranqulidad puede cuantificarse objetivamente a través del coste del arrendamiento de una vivienda similar, a lo que habría que añadir la cuantificación de los elementos que de cada supuesto deriven: por ejemplo, la necesidad de soportar mayores costes de desplazamiento al lugar de trabajo u ocio y el mayor tiempo invertido en ello. De haberse optado por el cambio temporal de residencia, habrá de aplicarse además un porcentaje derivado de la necesidad de abandonar el propio domicilio, que la sentencia propone objetivar a través de la previsiones de la 
legislación de expropiación forzosa relativas al valor de afección. En los supuestos en los que no haya cambio temporal de residencia, bien porque se carece de medios para ello, bien porque, de tenerlos, se establece por los interesados un orden de prelación de intereses y gastos contrario al traslado, ello no significa ni la ausencia del daño, ni la aceptación del mismo, ni que no deba ser resarcido. La objetivación del daño puede tener lugar a través del coste de un arrendamiento similar y a ello habría que añadirle la indemnización por el daño moral derivado de soportar la incomodidad. $Y$ aquí quizás se pudiera aplicar el porcentaje de menor valor que las viviendas ubicadas en zonas sometidas a ruido presenten respecto de las similares ubicadas en zonas ajenas a tales molestias. De no haberlas el coeficiente aplicable debería ser de naturaleza subjetiva.

\section{SANCIONES ADMINISTRATIVAS POR OCUPACIÓN DEL DEMANIO COSTERO Y POR ACAMPADA EN PLAYAS}

En relación a la sanción impuesta a una asociación por la ocupación sin título de más de ciento cuarenta metros cuadrados de dominio público marítimoterrestre mediante toldo, postes, anclajes y diverso mobiliario en dos terrazas, en el municipio de Lekeitio, la STSJPV 495/2017, de 29 de diciembre (sala de lo contencioso-administrativo, sección primera, ponente: Murgoitio Estefanía), anula la resolución sancionadora en base a diferentes argumentos. En primer lugar, se plantea la duda de la condición de tercero hipotecario de la asociación sancionada. Esta condición es importante pues de existir la misma tendría derecho a una concesión compensatoria de ocupación del demanio. Es cierto que consta en el expediente que quien tiene inscrita a su nombre la finca es una sociedad anónima diferente de la asociación mencionada. Pero no lo es menos que la citada asociación la tuvo inscrita a su nombre hasta junio de 1978, como consecuencia de una permuta con el Ayuntamiento, que había inmatriculado la finca a su favor en 1970. Que dicha inicial inmatriculación fuera nula, como alegaba la Abogacía del Estado y que, por ende, la asociación nunca hubiera debido alcanzar la condición de tercero hipotecario es algo que no puede dilucidarse en este proceso, en el que solo se cuestiona la legalidad de la sanción administrativa impuesta, sino que debería producirse mediante el ejercicio de las accciones judiciales pertinentes para la invalidación del título en 
la jurisdicción civil. Llegados a este punto, y sin perjuicio de que el expediente sancionador podría haberse dirigido no contra la asociación sino contra la sociedad mercantil que constaba en ese momento como titular registral, la solución es clara para el tribunal. Quien detenta una apariencia de derecho a la ocupación, no ya por el largo periodo temporal de ejercicio de la misma (se habla de más de sesenta años), sino por contar con la protección y presunción de detentación legítima que inicialmente se deriva del Registro de la Propiedad, no puede ser válidamente inquietado en ella por medio de acciones administrativas punitivas, al menos si con ese amparo residual ha emprendido ya antes, y ante la propia administración sancionadora, la actividad propia de legalización y transformación de su derecho que ante esta última pende, y cuando esa iniciativa debía conducir con alta previsión racional a la confirmación de ese derecho a la ocupación.

La STSJPV 346/2017, de 18 de septiembre (sala de lo contenciosoadministrativo, sección primera, ponente: Murgoitio Estefanía), declara la disconformidad a Derecho de una sanción administrativa de doscientos cuarenta euros impuesta por acampada ilegal en la playa de Laga (municipio de Ibarrangelua). El órgano judicial considera que imponer una multa en un supuesto de hecho tan conjetural, incierto e hipotético como el que presenta este caso no cuenta con el debido fudamento. $Y$ es que la base de la sanción impuesta es la constatación por agentes de la Guardia Civil del aparcamiento de una furgoneta a las siete y veinte de la mañana, en la que se aprecia una persona durmiendo en su interior, en una zona en la que existe un aparcamiento veraniego de más de dos mil metros cuadrados, que efectivamente- según el deslinde forma parte del demanio marítimo-terrestre, pero que se halla en una zona asfaltada, en la que se aprecian bocas de redes subterráneas de suministro o saneamiento, que se halla delimitada por postes y cables como cierres y que difícilmente puede entenderse incluida en el ámbito de la playa, sino más bien situada en una zona aledaña a la misma. El vehículo estacionado carece de las características de las autocaravanas, no ha desplegado elementos adicionales de prolongación (toldos, mesas, techos elevados, etc.) que revelen la intención de acampar y, además, el hecho de 
que en su interior hubiera una persona durmiendo no puede considerarse pernoctación (reposo a lo largo de toda la noche).

\section{ILEGALIDAD DE SANCIÓN IMPUESTA A CENTRO DE SALUD PÚBLICO POR FALTA DE COMUNICACIÓN PREVIA DE ACTIVIDAD CLASIFICADA}

En la STSJPV 521/2017, de 13 de noviembre (sala de lo contenciosoadministrativo, sección segunda, ponente: Ruiz Ruiz), el órgano judicial estima el recurso de apelación interpuesto por Osakidetza-Servicio Vasco de Salud contra tres sanciones administrativas de multa de seis mil euros, impuestas por el Ayuntamiento de Portugalete, por infracción leve prevista en la Ley General de Protección del Medio ambiente del País vasco, debido a que dicha entidad pública autonómica comenzó a operar un centro de salud sin previa comunicación de actividad clasificada al Ayuntamiento de Portugalete. El TSJPV revoca la sentencia apelada (que había dado la razón al Ayuntamiento) y declara la nulidad de las resoluciones sancionadoras impuestas al considerar que en este ámbito sancionador en el que nos movemos y en un supuesto tan singular como el presente, no puede utilizarse el procedimiento sancionador, por ausencia de comunicación formal previa, cuando ha de concluirse que el Ayuntamiento, esto es las autoridades municipales, fueron directamente conocedoras de la puesta en funcionamiento del centro de salud, debiéndose recalcar la diferencia de las potestades ordinarias en el ámbito de las actividades y las singulares en las que se desenvuelve el derecho sancionador. El órgano judicial considera, en esa línea, varios factores como relevantes para justificar su decisión: la singularidad de que se estén enfrentando en este procedimiento sancionador dos organismos públicos, el dato de que se sancione por falta de comunicación previa la apertura de un centro de salud en cuanto que servicio esencial cuya existencia promovió el propio Ayuntamiento y fue objeto de inauguración oficial, y que se comunicaran, mediante un requerimiento municipal, los presupuestos a cumplimentar a efectos del cumplimiento de las exigencias de la normativa de actividades clasificadas por parte del director de urbanismo del Ayuntamiento (no por el alcalde, persona competente en esta materia) y, además, sin ofrecimiento de la vía de recurso contra ese requerimiento. 


\section{GASTOS DE DESCONTAMINACIÓN DEL SUELO Y REPARCELACIÓN URBANÍSTICA}

La STSJPV 495/2017, de 31 de octubre (sala de lo contencioso-administrativo, sección segunda, ponente: Alberdi Larizgoitia) afirma que los gastos de descontaminación de suelo contaminados de un ámbito reparcelatorio corresponden en primer lugar y de modo principal al causante de la contaminación y, subsidiariamente, a los propietarios o poseedores por dicho orden, y sólo adquieren la consideración de cargas de urbanización cuando justificadamente el Ayuntamiento ordene a la comunidad reparcelatoria la ejecución de la descontaminación y adecuación de los terrenos, lo que tiene como presupuesto que no sea posible la descontaminación con cargo al obligado principal. 